\title{
A chance encounter changes everything
}

\author{
Vijay Sankaran is a practicing pediatric hematologist and researcher at Boston Children's Hospital, the Dana-Farber \\ Cancer Institute and the Broad Institute of MIT and Harvard. His laboratory uses insights from human genetics to \\ study blood cell production in health and disease.
}

\author{
Vijay G. Sankaran
}

still vividly remember the day I met Robert $^{\star}$ when I was shadowing in a hematology clinic as a first-year medical student in Harvard's MD-PhD program. Prior to beginning medical school, I hadn't spent much time in hospitals and only had limited exposure to the clinical realm, which fueled my desire to learn more about what the world of medicine was like. I was also at a crossroads in what to do as a graduate student. My prior research was in biochemistry and structural biology, but I realized that broader physiological questions greatly appealed to me. Looking back, that meeting with Robert had a profound impact on me and would forever change my focus as both a physician and scientist.

At 24 years old, Robert was slightly older than me. For the first 17 years of his life, he had frequently been hospitalized with pain and other complications due to sickle cell disease. Then, things changed dramatically. Robert had started hydroxyurea, a medication that stimulates the production of a fetal form of hemoglobin and can thereby reduce clinical symptoms in individuals with sickle cell disease. I sat in the clinic talking to Robert about his experiences while the attending physician I had been shadowing hurriedly ran to see a patient in another room. I was in awe at the remarkable effect that hydroxyurea had upon Robert, which forever changed his life.

As I learned more about hydroxyurea and the regulation of fetal hemoglobin, I realized that, while hydroxyurea was incredibly beneficial for some patients, like Robert, many patients with sickle cell disease who received this treatment continued to have clinical symptoms, including frequent episodes of severe pain. Studies had shown that greater fetal hemoglobin production would result in an improved clinical course in sickle cell disease. I spent a couple of weeks reading and studying numerous papers describing what was known regarding the regulation of fetal hemoglobin. I realized that, despite decades of work, lots of scientific questions remained unanswered. This scientific problem and the potential for clinical impact sparked my interest.

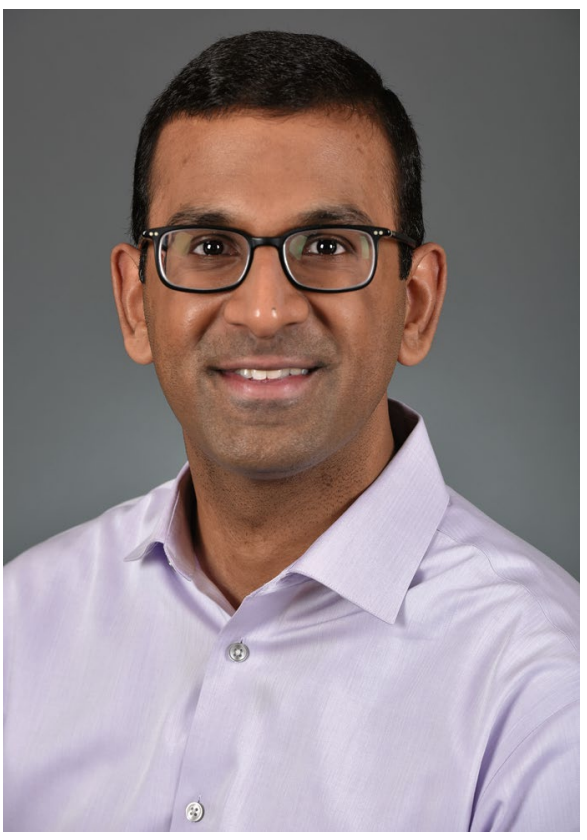

Credit: Katherine Cohen, Boston Children's Hospital

Around the same time that I met Robert, I had been working in a very prominent laboratory, and I loved the scientific environment. However, I was not in love with the scientific problem I was pursuing (likely due to my own naivete at the time): how stem cells could remain in their pluripotent state. As soon as I started to read about the work on the regulation of fetal hemoglobin, much of which had been done decades earlier in the same lab I was working in, I went to the principal investigator and told him that I was no longer interested in the problem in stem cell biology he had suggested I work on. Instead, I wanted to study fetal hemoglobin regulation. I am sure many principal investigators would have shown me out the door, but I was fortunate to get a very different reception. The principal investigator, Stu Orkin, who continues to be a close mentor and friend, said: sure, why don't we give it a shot?

I spent a couple of years failing in my attempts to pursue this problem, but I nonetheless learned a tremendous amount. By the time I got into my third year in the laboratory, we had gathered DNA samples from patients with sickle cell disease, which, coupled with significant advances in human genetics in the preceding years, allowed us to be among the first involved in genetic studies on fetal hemoglobin regulation. These studies, which built upon work by Swee Lay Thein and the late Antonio Cao, led us to a gene known as BCL11A. Having unsuccessfully worked on fetal hemoglobin regulation for years, we were well set up to start dissecting the role of BCL11A and were able to demonstrate that it is critical for silencing fetal hemoglobin. This work opened up the possibility of developing drugs or other targeted therapies that act on BCL11A to help patients with sickle cell disease produce more fetal hemoglobin.

This pivotal experience taught me three things. First, I realized the immense value that studies of human genetic variation can have in gaining insights into biological problems - something that drives the research we do in my own laboratory now. Second, I realized that I truly love being able to work on scientific problems inspired by the patients I am fortunate to care for clinically. Third, I realized the enormous value of giving freedom to trainees to pursue problems that stimulate them, which is an important principle I put into practice in my own laboratory now. Meeting Robert and having the chance to pursue a scientific problem motivated by this clinical encounter forever changed my career, as a physician and as a scientist.

${ }^{*}$ Patient name has been modified to avoid identification.

\footnotetext{
Vijay G. Sankaran 1,2,3

${ }^{1}$ Division of Hematology/Oncology, Boston Children's Hospital, Harvard Medical School, Boston, MA, USA. ${ }^{2}$ Department of Pediatric Oncology, Dana-Farber Cancer Institute, Harvard Medical School, Boston, MA, USA. ${ }^{3}$ Broad Institute of MIT and Harvard, Cambridge, MA, USA.

e-mail: sankaran@broadinstitute.org
}

Published online: 6 June 2019 https://doi.org/10.1038/s41591-019-0418-2 